\title{
Metallic powder flowability: a comparison between testing methods and the effect of particle size distribution
}

\author{
Lorenzo Marchetti1, Christopher Hulme-Smith1 \\ KTH - Royal Institute of Technology. Department of Materials Science and Engineering
}

\begin{abstract}
The flowability of a powder is a characteristic dependent on both the particle ensemble's physical properties as well as the testing equipment and conditions. In powder bed additive manufacturing processes (SLM, EBM), flowability can be used to predict the quality of the deposited powder layer. However, how representative flowability is for the powder bed layer quality is not always clear.

In this work, we compared several steel powders, as well as different testing methods. Each powder had an individual combination of composition or particle size distribution. Furthermore, the flowability testing equipment and methods were selected according to standards (Tap density, Hall flow) or referring to the published literature (angle of repose, FT4 Powder Rheometer). After measuring the flowability of different samples for each testing method, we created a database to evaluate a first correlation between the different testing methods. Finally, with a Powder Rheometer, we investigated for one sample some flow properties as function of different particle size distributions.
\end{abstract}

\section{Keywords}

Flowability, Powder rheometer, Powder characterization

\section{Introduction}

The study of the flow behaviour of powder and the associated testing methods is a challenging topic which affects different research fields, from powder metallurgy and pharmaceuticals to food production. The current rapid growth of interest from industries and researchers in metal powder-based additive manufacturing has highlighted the need to ensure powders are suitable to ensure good-quality final components [1-4]. One prime example is the ability to form an even, thin and compact layer of powder in powder bed based processes. However, there is yet no agreement on the best method to describe powder's flowability (or spreadability) for powder bed-based additive manufacturing or any other process [5-10]. It is also not clear how the powder physical characteristics can affect flowability [5, 7, 11]. This lack of agreement has been attributed to the complex multivariable nature of powder flow [5].

Powder flowability is a characteristic that describes the dynamic behaviour of a bulk powder sample. At a fundamental level, flow behaviour depends on the balance between forces that promote the motion of powders and those that prevent it. Forces that promote flow can include gravity and applied external forces. Forces opposing flow include frictional forces (both between the powder particles and external surfaces and between two individual powder particles), mechanical interlocking of particles and other forces between neighbouring powder particles (e.g. van deer Waals forces, liquid bridging forces, electrostatic forces and magnetic attraction) [12]. The finer the powder, the more points of contact there are between particles 
and so the forces between particles are more significant. Processes that use fine powders include metal injection moulding and additive manufacturing. These high inter-particle forces can make the powder cohesive under certain conditions and impede or prevent powder flow. This could prevent, for example, the deposition of a uniform layer during deposition of a new powder bed layer in an additive manufacturing process $[9,11]$. A non-uniform powder layer has been proven to affect the quality of the final component $[4,13,14]$.

These forces will depend on several physical variables, some of which related to the powder, for example particle size and size distribution, particle shape, material density, particles roughness $[6,15]$. Other forces may also arise from the testing equipment, such as friction between the powder particles and the equipment surfaces. Like all measurements of time-dependent properties, the testing method and conditions (e.g. static or dynamic testing, powder stress state, powder humidity, temperature, eventual gas flow) also affect the behaviour observed in test.

Due to the multivariable nature of the powder flow and the large number of discrete bodies involved, it is often argued that building a theoretical model to describe it would be challenging, if not practically impossible[5]. Models published in literature are complex or restricted to some specific case and they don't explicitly include physical variable $[9,16]$. Therefore, the flow behaviour of powders is often characterized and studied through comparative testing. In order to obtain significant results, it is generally suggested that the testing conditions should be as close as possible to the real case conditions. However, in powder bed additive manufacturing, where metallic powders are spread on a thin layer, testing the spreadability of the powder is not well-established. One possible explanation is that the evaluation of the spread layer is challenging and ill-defined. Other common flowability testing methods are used but are often argued to not be fully representative of the powder spreading conditions during the process.

The presence of several different flowability testing methods gives the possibility to study the powder flow in different conditions. Some of these testing methods are standardized, such as the Hall flowmeter and Carney flowmeter [17, 18]. However, there is not a clear description of which method should be adopted for a specific application [19]. As a further matter, the methods proposed in the ASTM standards present a stress state which is different from the one in additive manufacturing and so measurements may not be useful. In the published literature it is possible to find other methods to assess the flowability of powders, including tap density testing, static and dynamic angle of repose, rheometry and shear cell tests) $[6,9]$. Authors do not always agree on the best technique to use $[6,9,20]$ and some common methods found in literature are not standardised, such as dynamic angle of repose and rheometry $[6,9,21]$. Hence, different studies may use different testing methodologies to make the same assessment and results may not be comparable.

Comparisons of flow behaviour in metal powders have been published, focusing either on the powder physical properties and on the different testing methods $[1,4,6,7,16,22]$. Some studies found correlation between powder properties and flowability $[4,6,9,16]$ and others found correlation between different flow measuring techniques $[1,9,16]$. However, studies exist that found no statistically-significant correlations [7, 22]. Most of these studies assessed linear relationships between the test variables $[7,16]$.

In the present work we compare several powder flowability measuring techniques on different steel powders designed for additive manufacturing. The current focus is on the flowability measuring techniques. The work is ongoing and data acquisition using more materials and measurement techniques is seen as a continuous process. The results presented here represent the end of the first phase of research. The same statistical tests will be applied continually as more data become available. 


\section{Experimental methods}

In this work we tested a range of different steel powders with several flowability testing methods. The flowability tests were adopted following the standards [17-19, 23] and other relevant literature [1, 21, 24].

\section{Materials and sampling}

First the flow properties of eleven steel powders from different powder producers were measured. Ten of these powders are designed for additive manufacturing, while one for hot isostatic pressing (Table 1).

In second place, one single powder was sieved to obtain four different size ranges. Selected finer and coarser size ranges were then re-combined to into 2 sets of mixtures, each made of 5 samples with different ratios of fine and coarse particles (Table 2).

Table 1. Powders used in the comparative flowability experimental trials. Powde \#6 is designed for hot isostatic pressing and all others for AM.

\begin{tabular}{cccc}
\hline Sample & Alloy & $\begin{array}{c}\text { Atomization } \\
\text { process }\end{array}$ & $\begin{array}{c}\text { Size range }[\mu \mathrm{m}] \\
\text { est. }\end{array}$ \\
\hline 1 & $17-4 \mathrm{PH}$ & Water & $<75$ \\
2 & $316 \mathrm{~L}$ & Water & $<75$ \\
3 & $17-4 \mathrm{PH}$ & Gas & $<15$ \\
4 & $17-4 \mathrm{PH}$ & Gas & $10-53$ \\
5 & $17-4 \mathrm{PH}$ & Gas & $63-106$ \\
$6 *$ & $316 \mathrm{~L}$ & Gas & $<53$ \\
7 & $316 \mathrm{~L}$ & Gas & $15-53$ \\
8 & 420 & Gas & $<53$ \\
9 & Tool steel 1 & Gas & $20-70$ \\
10 & Tool steel 2 & Gas & $20-70$ \\
11 & Tool steel 3 & Gas & $15-70$ \\
\hline
\end{tabular}

Table 2. Samples used to study the effect of different particle size distributions on flowability. All the samples were sieved from the same batch of AISI 420.

\begin{tabular}{cccc}
\hline Crmins & $\begin{array}{c}\text { Finer mass } \\
\text { fraction }[\%]\end{array}$ & $\begin{array}{c}\text { Finer size range } \\
{[\mu \mathrm{m}]-\text { est. }}\end{array}$ & $\begin{array}{c}\text { Coarser size range } \\
{[\mu \mathrm{m}]-\text { est. }}\end{array}$ \\
\hline 21 & 0 & & $40-56$ \\
22 & 25 & $0-20$ & \\
23 & 50 & & \\
24 & 75 & & \\
25 & 100 & & \\
26 & 0 & & \\
27 & 25 & & \\
28 & 50 & & \\
29 & 75 & & \\
30 & 100 & & \\
\hline
\end{tabular}


Each powder was sampled with a rotary riffler in accordance with the applicable standards [25]. After being divided, samples were then dried for $30 \mathrm{~min}$ at $105-110^{\circ} \mathrm{C}$ before being tested. This reduced the total moisture content and ensured all powders had a similar moisture content

\section{Flowability comparative testing}

The flow properties of the samples used in the comparative flowability tests (Table 1, samples 1-11) were characterized with 8 different techniques (Table 3). Some of these are traditionally meant to characterize flowability. The remaining testing methods are usually adopted to characterize other behaviours involving the motion of powders, which can in turn be related to the powder flow properties.

Generally, the testing of flowability has been carried out using funnel methods. A fixed mass of powder is allowed to flow through a conical funnel with a fixed geometry while the flow time is recorded. Up to today, three standardised funnel geometries can be found: Hall [26], Carney [17] and Gustavsson [27]. The Hall funnel has an opening of $2.5 \mathrm{~mm}$ and a cone angle of $60^{\circ}$. Cohesive powders can't flow through the Hall funnel and are run through the Carney funnel, which has a wider opening $(5 \mathrm{~mm})$. Also the Gustavsson flow funnel is designed for cohesive powders: it has an opening of $2.5 \mathrm{~mm}$, but a steeper cone angle of $30^{\circ}$. Time is recorded manually, making the funnel methods easily subjected to systematic errors. In this work, we characterized flowability with Hall and Carney funnels.

The angle of repose was determined with the fixed height funnel method. Powder samples of $25 \mathrm{ml}$ were poured through a funnel on a static plate. The angle at the base of the cone of powders is the angle of repose. This angle is said to describe the inter-particle friction, which strongly affects flowability [16]. This method relies on the operator correct execution both pouring the powder and in manually reading the angle.

Tapped density was measured as volume per weight after mechanically tapping the sample with a standardised machine. The tapped density is an indicator of how well a powder can rearrange when mechanically agitated. It is often considered as an indication of flowability [28] through the Hausner Ratio $(H)\left(\left(1\right.\right.$, where $V_{0}$ is the volume of the as-poured powder and $V_{f}$ is the volume of the same sample after tapping).

$$
\mathrm{H}=\frac{\mathrm{V}_{0}}{\mathrm{~V}_{\mathrm{f}}}
$$

A Freeman FT4 Powder Rheometer was used to run several pre-set tests. In the flowability test sequence an impeller moves through the powder contained in a cylindrical vessel following a pre-set path, while the resistance to its movement is recorded. The test involves several runs in different conditions, varying the blade tip speed and considering the upward and downward blade movement. Some powder flow properties, such as Basic Flowability Energy (BFE) and the Specific Energy (SE), are automatically derived. BFE is the energy required to move the blade downwards through the sample (high stress mode, where the powder is moved while subjected to a confined stress against the lower part of the vessel), while SE is the specific energy (over mass) required to move the blade upwards (low stress mode, where the powder are moved in an unconfined stress state). The higher the energy required to move the powder, the lower the flowability $[10,21]$.

In the rheometer compressibility test sequence powders are compressed within a cylindrical vessel using a flat disc that fits precisely the vessel. The applied stress cycle is preset, while the change in the powder column height is recorded. The compressibility (CPS), which is the percentage volumetric variation at $15 \mathrm{kPa}$, is then automatically derived. 
Although compressibility is meant to describe the ease of a powder being compressed at low stress, it can relate to flowability, since the particles must move past each other to allow the volume of the ensemble to change.

In the shear cell test sequence the powder is sheared under an applied normal compressive stress (consolidating stress). The powder is first compressed under a simple uniaxial stress with pre-defined magnitude. The shear stress is then gradually increased until the onset of flow is reached. The shear and normal stresses at this point are recorded. This is repeated with a range of compressive stresses applied at the same time as the shear stress. In between every repetition the condition of the powder is reset by pre-shearing it under the initial consolidating stress, until the powders flow in a steady state condition. The yield locus for a given consolidating stress is then plotted on a cr- $\mathrm{T}$ diagram, where the shear stress required to initiate flow at different compressive stresses is shown. The results are commonly summarized through different output data:

- Cohesion: describes the shear stress required to start the flow when the normal stress is zero.

- $\quad$ UYS (Unconfined yield stress): the normal equivalent stress required to cause the onset flow when the powder is simply compressed in one direction.

- $\quad$ MPS (major principal stress): the normal equivalent stress required to maintain steadystate flow.

- $\quad F F$ (Jenike's flow function): $F F=M P S / U Y S$. A high value indicates that it is significantly easier to maintain flow than to initiate it.

- $\quad$ AIF (Angle of internal friction): represents the angle of the yield locus.

All the rheometer tests were performed twice with two representative specimen of the same sample. The shear cell test sequence was repeated with a consolidating stress of $3 \mathrm{kPa}$ and $9 \mathrm{kPa}$. This to investigate the the effect of the compressive loading on the shear properties.

\section{Flowability comparative testing correlations}

The samples (Table 1, samples 1-11) used in the comparative flowability tests (Table 3) were recorded into a dataset comprehensive of different flow and powder properties, which is being created. In this study, an overview of the relationships between the so far performed flowability measurements is presented.

A matrix correlation analysis was performed using the Pearson coefficient between the flow properties of all the powders tested. The Pearson coefficient is a measure of the strength of the linear correlation between two variables. To permit this analysis to be used, it is implicitly assumed that the data are normally distributed and linearly dependent. Moreover, each pair of variables is assessed independently from the other variables. At this stage physical properties and flow metrics are treated in the same way.

It is possible to begin to understand the intricate relationship between the different flowability testing methods. This will be used to direct our future investigations.

\section{Flowability comparative testing shortcomings}

In the comparative flow testing analysis there are four main shortcomings:

- $\quad$ Powders have been dried and tested right after, but humidity hasn't been measured. Finer powders will adsorb humidity faster, possibly leading to a slightly different behaviour. 
- $\quad$ Some flowability testing methods are not included in the present work. This includes the dynamic angle of repose $[6,9,24]$ and the Gustavsson flowmeter. Both methods have been found suited for AM powder flowability characterization.

- $\quad$ More powders are needed in order to produce a more robust statistical analysis.

Further experiments are planned to collect these data.

\section{Flowability and particle size distribution}

The effect of different particle size distribution on flow properties have been tested on one material. One specimen for each sample (samples 20-30, Table 2) underwent the rheometer flowability test sequence. The values representing some flow properties (BFE and $\mathrm{SE})$, as well as the conditioned bulk density (CBD), were then recorded and compared.

Table 3. Overview of the methods performed. The motion state indicates whether the powders are tested more in dynamic or static conditions. The stress state indicates if the powders are tested under the effect of some stress (gravity, applied shear or normal stresses) that can influence their flow behaviour. The operator influence points out that for some tests the skills of the operator might influence the measurements.

\begin{tabular}{|c|c|c|c|c|c|}
\hline $\begin{array}{c}\text { Testing } \\
\text { equipment }\end{array}$ & Test performed & $\begin{array}{l}\text { Measured } \\
\text { variable }\end{array}$ & $\begin{array}{l}\text { Motion } \\
\text { state }\end{array}$ & $\begin{array}{l}\text { Stress } \\
\text { state }\end{array}$ & $\begin{array}{c}\text { Operator's } \\
\text { influence }\end{array}$ \\
\hline Hall flowmeter & Flowability & Flow time $[\mathrm{s}]$ & Dynamic & Low & High \\
\hline Carney funnel & Flowability & Flow time $[\mathrm{s}]$ & Dynamic & Low & High \\
\hline Angle of repose & Angle of repose & Angle $\left[{ }^{\circ}\right]$ & Static & None & Very high \\
\hline Tap-meter & Tapped density & Hausner Ratio [-] & Dynamic & Low & Average \\
\hline \multirow{3}{*}{ Rheometer } & Clo & $\mathrm{BFE}[\mathrm{mJ}]$ & Dynamic & Average & \multirow{3}{*}{ Low } \\
\hline & THowatity & $\mathrm{SE}[\mathrm{mJ}]$ & Dynamic & Low & \\
\hline & Compressibility & CPS@15 kPa & Static & High & \\
\hline \multirow{10}{*}{$\begin{array}{l}\text { Shear cell } \\
\text { (Rheometer) }\end{array}$} & \multirow{5}{*}{ Shear cell $3 \mathrm{kPa}$} & Cohesion & \multirow{5}{*}{ Static } & \multirow{5}{*}{ Average } & \multirow{10}{*}{ Low } \\
\hline & & UYS [kPa] & & & \\
\hline & & MPS $[\mathrm{kPa}]$ & & & \\
\hline & & FF & & & \\
\hline & & $\mathrm{AIF}\left[^{\circ}\right]$ & & & \\
\hline & \multirow{5}{*}{ Shear cell $9 \mathrm{kPa}$} & Cohesion & \multirow{5}{*}{ Static } & \multirow{5}{*}{ Average } & \\
\hline & & UYS [kPa] & & & \\
\hline & & MPS [kPa] & & & \\
\hline & & $\mathrm{FF}$ & & & \\
\hline & & $\operatorname{AIF}\left[^{\circ}\right]$ & & & \\
\hline
\end{tabular}




\section{Results and discussion}

\section{Flowability comparative testing correlations}

Figure 1 shows two charts where the correlation matrices obtained from the different flowability tests performed are plotted. The first chart shows all the linear correlation coefficients obtained. The second chart (to the right) shows the correlation coefficients filtered according to their significance ( $\mathrm{p}$ value $<0.05$, or $2 \mathrm{cr}$ ).

The first chart shows many correlations between many of the testing variables. Some correlations results stronger, some weaker. It is notable to observe some strong correlation between the basic flow energy (BFE), specific energy (SE) and the Hall flow time. These however are not so strongly related to a second group of parameters, composed form the angle of repose (AOR), Compressibility (CPS), and conditioned bulk density (CBD). The Hausner ratio seems to sit in between these two group of variables. Finally several other correlations occur between the many parameter that describe the shear cell analysis. From this, it might be deduced that some parameters, such as BFE or SE, might be deduced from less expensive methods, such as Hall flow time. Conversely, other parameters, such as the angle of repose (AOR) might describe aspect of the powder flow in different conditions, and could be complementary.

However, the second chart, where the results were filtered to exclude those for which the significance level $\mathrm{p}$ was less than 0.05 , shows still some correlations. Here more correlations would be expected in the lower right section of the chart, representing the area containing the shear cell variables, as all these metrics come from the same test group. The results suggest that the angle of repose (AOR) is directly related to the Hausner ratio and the compressibility (CPS) inversely proportional to the conditioned bulk density (CBD). This could suggests that the powder flow in these three tests is limited by the same mechanism. Further investigation would be required to positively identify this mechanism. On the other hand, it is suggested that the specific energy (SE) is poorly related the angle of repose (AOR), but more to the Hausner ratio.
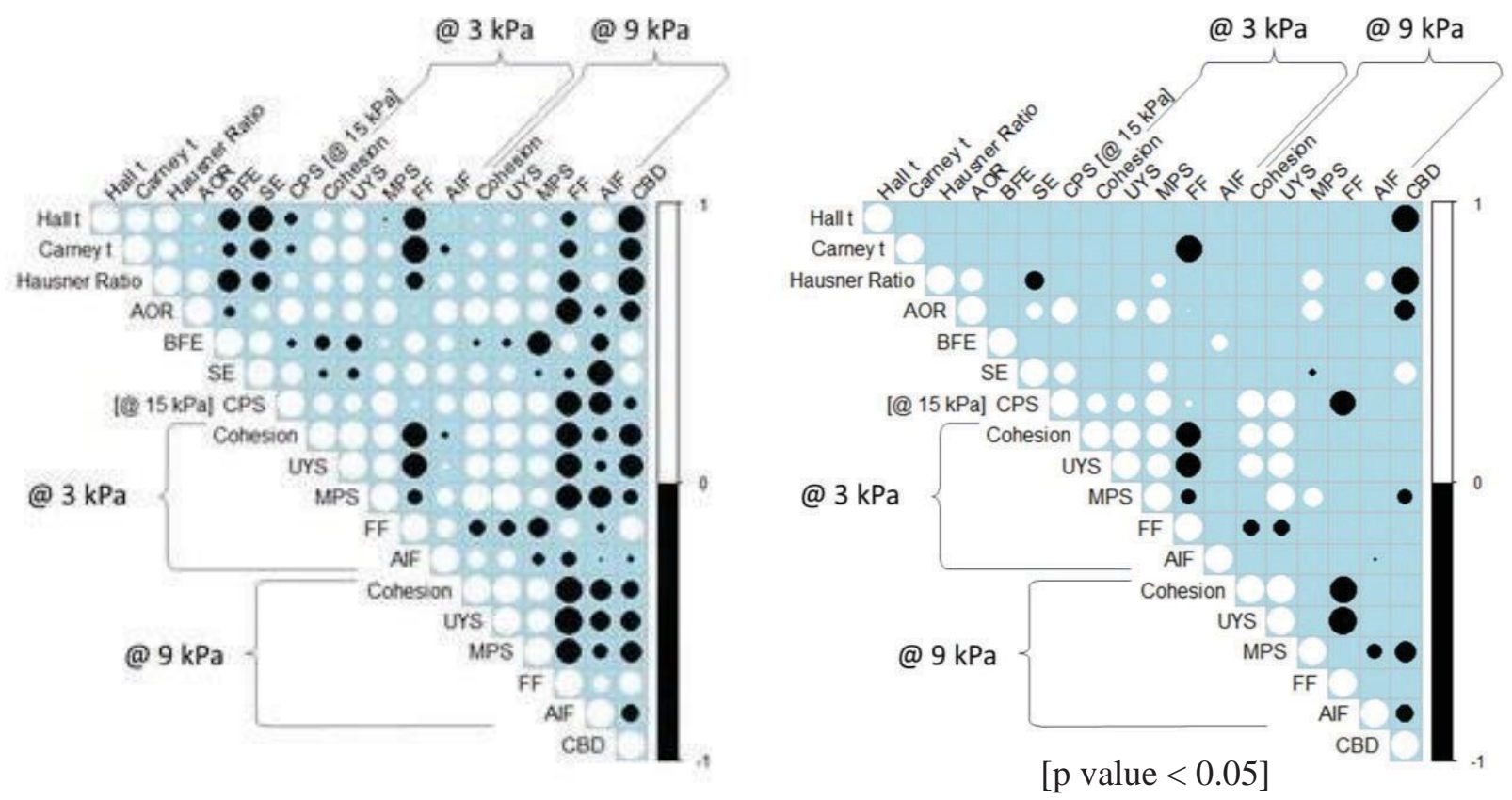

Figure 1. Correlation matrices of the different flow properties. The size of the circles represents the strength of the linear relationship (Pearson coefficient from 0 to 1 ). The 
colour describes the direction of the linear relationship (white for a positive Pearson coefficient, black for a negative one). The chart on the left shows all the Pearson correlation coefficients, independently on how well they fit the data. The chart on the right was been filtered to show only the significant relationship between flow properties ( $\mathrm{p}<$ 0.05 , or $2 \mathrm{cr}$ ). The conditioned bulk density (CBD) recorded with the rheometer was added to the correlation matrices.

\section{Flowability and particle size distribution}

Figure 2 shows two charts where powder properties are represented in function of the mass fraction of fine particles added to the powder mixes. In figure $2 \mathrm{~A}$ it is possible to observe the increase in the conditioned bulk density (CBD) when combining finer and coarser particles, as expected. The two powder mixes behave similarly, with some deviation when $25 \%$ in mass of fine powder is added to the powder mix. In figure $2 \mathrm{~B}$ is shown the basic flow energy (BFE) in function of the mass fraction of finer particles added to the powder mix. Here the flow behaviours shown are different: even if the addiction of finer powder always leads to a significant decrease of BFE, the trend is quite different. For the powder mix consisting of the fractions $0-20$ and $40-56 \mu \mathrm{m}$ the drop is linear, while for the mix consisting of the fractions $20-40$ and $56-71 \mu \mathrm{m}$ the trend drop is non-linear and seems leading to a plateau.

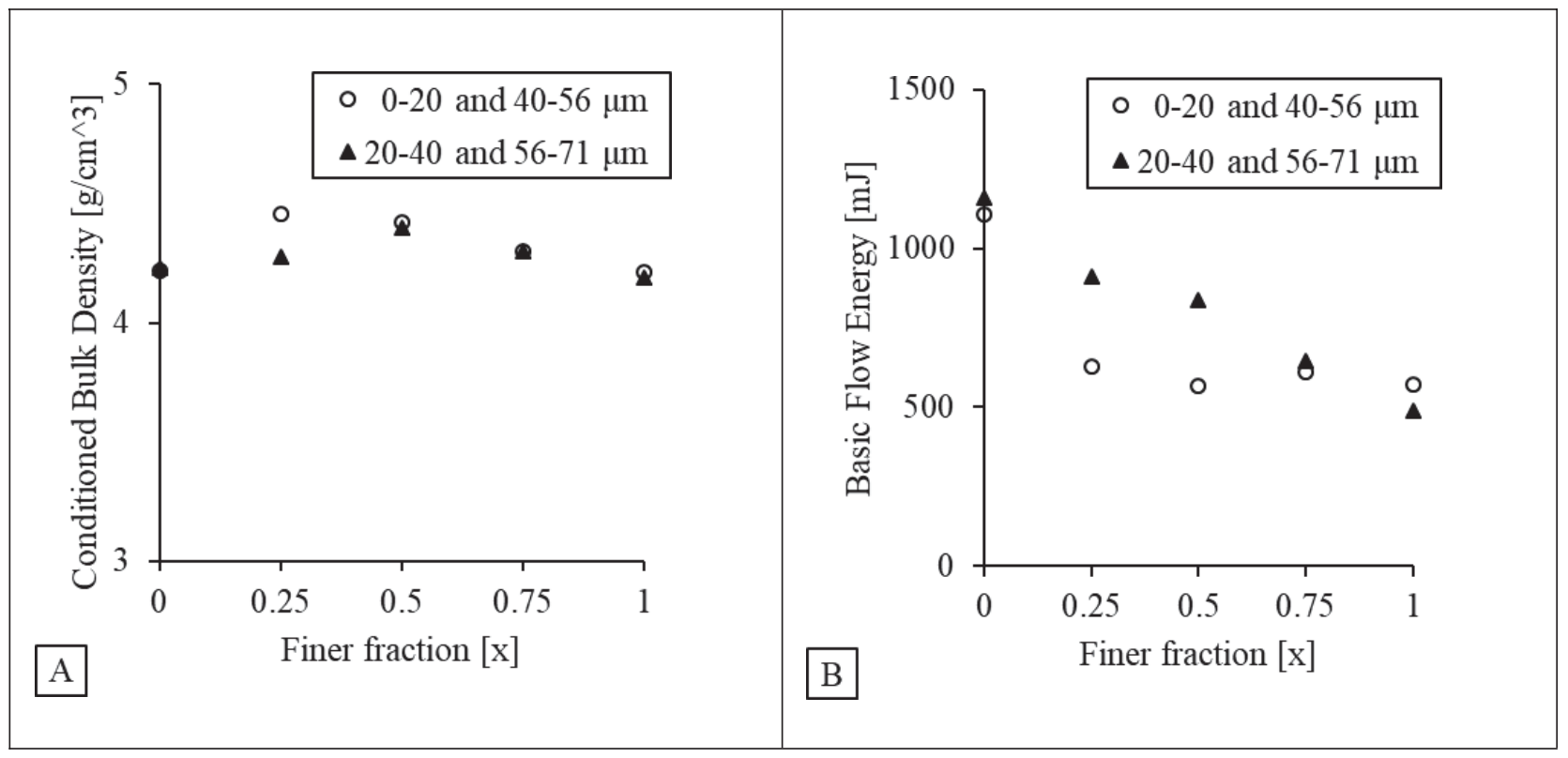

Figure 2. Conditioned Bulk Density (CBD) in function of the mass fraction of finer particle for the two powder mixes tested (A). Basic Flow Energy (BFE) in function of the mass fraction of finer particle for the two powder mixes tested (B).

\section{Conclusions}

Regarding the correlations between different flowability testing methods, in the set of powders tested, it was possible to find correlations between different flowability measurements:

- $\quad$ Angle of repose (AOR), compressibility (CPS) and partly Hausner ratio were found to be positively correlated to each other. Hausner ratio and angle of repose (AOR) were also negatively correlated to the conditioned bulk density (CBD). 
- $\quad$ Some relationship between the former group of variables with the specific energy (SE), but not very strong and with opposite sign.

More powders and physical properties must be tested, both to confirm and explain the trends already observed and to explore new correlations. This forms the upcoming plan for future work in this research.

Regarding the influence of the different particle size mixtures on the basic flowability energy (BFE) it is observed that it is possible to achieve a higher conditioned bulk density (CBD) by mixing a fine and coarse powder fraction (finer mass fraction in between 0.25 and 0.50). Moreover, for these densities the Basic flow energy (BFE) indicates intermediate properties between coarser and finer powder. However, to better define this trend further investigations are required.

\section{Bibliography}

[1.] Gibson, I., Rosen, D. W., and Stucker, B., Additive Manufacturing Technologies: 3D Printing, Rapid Prototyping, and Direct Digital manufacturing, 2nd ed., Springer, New York, 2015, pp. 1-498.

[2.] Rajeev Dattani, K.B., Jamie Clayton, Optimising feedstock selection for Additive Manufacturing, in Euro PM2018. 2018: Bilbao.

[3.] Clayton, J., Optimising metal powders for additive manufacturing. Metal Powder Report, 2014. 69(5): p. 14-17.

[4.] Spierings, A.B., N. Herres, and G. Levy, Influence of the particle size distribution on surface quality and mechanical properties in AM steel parts. Rapid Prototyping Journal, 2011. 17(3): p. 195-202.

[5.] Prescott, On powder flowability. Pharmaceutical Technology, 2000.

[6.] Antequera, M.V.V., et al., Evaluation of an Adequate Method of Estimating Flowability According to Powder Characteristics. International Journal of Pharmaceutics, 1994. 103(2): p. 155-161.

[7.] Krantz, M., H. Zhang, and J. Zhu, Characterization of powder flow: Static and dynamic testing. Powder Technology, 2009. 194(3): p. 239-245.

[8.] Engeli, R., et al., Processability of different IN738LC powder batches by selective laser melting. Journal of Materials Processing Technology, 2016. 229: p. 484-491.

[9.] Spierings, A.B., et al., Powder flowability characterisation methodology for powder-bedbased metal additive manufacturing. Progress in Additive Manufacturing, 2015. 1(12): p. 9-20.

[10.] Leturia, M., et al., Characterization of flow properties of cohesive powders: A comparative study of traditional and new testing methods. Powder Technology, 2014. 253: p. 406-423.

[11.] Manfred Schmid, F.A. Flowability of SLS powders - a frequently neglected parameter. in 2014 Annual Additive Manufacturing Users Group Conference. 2014. Tucson, Arizona.

[12.] Berk, Z., Fluid Flow, in Food Process Engineering and Technology. 2018, Academic Press.

[13.] Vock, S.J., Solomon; Härtel, Michael; Klöden, Burghardt; Weißgärber, Thomas; Kieback, Bernd, A Systematic Approach for Understanding Powder Influence in Powder Bed Based Additive Manufacturing, in Euro PM2018. 2018: Bilbao. 
[14.] Dietrich, S., et al., A New Approach For A Flexible Powder Production For Additive Manufacturing. 16th Machining Innovations Conference for Aerospace Industry - Mic 2016, 2016. 6: p. 88-95.

[15.] Muthuswamy, M. and A. Tordesillas, How do interparticle contact friction, packing density and degree of polydispersity affect force propagation in particulate assemblies? Journal of Statistical Mechanics: Theory and Experiment, 2006. 2006(09): p. P09003-P09003.

[16.] Geldart, D., et al., Characterization of powder flowability using measurement of angle of repose. China Particuology, 2006. 4(3-4): p. 104-107.

[17.] ASTM, B964 - 16 Standard Test Methods for Flow Rate of Metal Powders Using the Carney Funnel. 2016.

[18.] ASTM, B213 - 17 Standard Test Methods for Flow Rate of Metal Powders Using the Hall Flowmeter Funnel. 2017.

[19.] ASTM, F3049 - 14 Standard Guide for Characterizing Properties of Metal Powders Used for Additive Manufacturing Processes. 2014.

[20.] Schulze, D., Powder and Bulk solids. 2007: Springer.

[21.] Freeman, R. and X. Fu, Characterisation of powder bulk, dynamic flow and shear properties in relation to die filling. Powder Metallurgy, 2013. 51(3): p. 196-201.

[22.] Mellin, P., et al., Evaluating flowability of additive manufacturing powders, using the Gustavsson flow meter. Metal Powder Report, 2017. 72(5): p. 322-326.

[23.] ASTM, B527 - 15 Standard Test Method for Tap Density of Metal Powders and Compounds. 2015.

[24.] François Lavoie, L.C.a.R.T., New Methods Characterizing Avalanche Behavior to Determine Powder Flow. Pharmaceutical Research, 2002.

[25.] ASTM, B215 - 15 Standard Practices for Sampling Metal Powders. 2015.

[26.] ASTM, B212 - 17 Standard Test Methodfor Apparent Density of Free-Flowing Metal Powders Using the Hall Flowmeter Funnel.2017.

[27.] ISO, Metallic powders - Determination of flowrate by means of a calibrated funnel (Gustavsson flowmeter) (ISO 13517:2013). 2013.

[28.] Santomaso, A., P. Lazzaro, and P. Canu, Powder flowability and density ratios: the impact of granules packing. Chemical Engineering Science, 2003. 58(13): p. 28572874. 